\title{
Selection of Wavelet Basis Function for Image Compression - A Review
}

\author{
Jaya Krishna Sunkara \\ Asst. Prof., Dept. of ECE, Sree Vidyanikethan Engineering College, A.Rangampet, India
}

Received $17^{\text {th }}$ June 2017; accepted 30 ${ }^{\text {th }}$ March 2019

\begin{abstract}
Wavelets are being suggested as a platform for various tasks in image processing. The advantage of wavelets lie in its time frequency resolution. The use of different basis functions in the form of different wavelets made the wavelet analysis a destination for many applications. The performance of a particular technique depends on the wavelet coefficients arrived after applying the wavelet transform. The coefficients for a specific input signal depends on the basis functions used in the wavelet transform. Hence, in this paper toward this end, different basis functions and their features are presented. As many image comprssion algorithms base on wavelet transform, selection of basis function for image compression should be taken with care. In this paper, the factors influencing the performance of image compression are presented. In addition to this, a broad review of wavelets in image processing applications and selection of basis function for different image processing tasks are presented.
\end{abstract}

Keywords: basis function, biorthogonal wavelets, image compression, vanishing moments.

\section{Introduction}

A computer is becoming more and more influential day by day. As a consequence of this, the use of digital images is growing briskly. Along with this increasing usage of digital images raises the serious concern of storing and transferring the gigantic volume of data representing the images. The reason behind this is that the uncompressed multimedia data entails substantial storage capacity and transmission bandwidth. Although there is a rapid progress in mass storage density, speed of the processor and the concert of the digital communication systems, the demand for data storage capacity and data transmission bandwidth remains to exceed the capabilities of on-hand technologies. Above and beyond, the latest growth of data intensive multimedia based web applications has placed much gravity on the researchers to discover the methods for using the images in the web applications more effectually. Internet teleconferencing, high definition television, satellite communications and digital storage of movies are unrealistic without a high degree of compression. Such applications are far from realizing their full potential largely due to the limitations of common image compression techniques [1]. The image is essentially a kind of redundant data i.e. it comprises the same information from certain perception of view. By using data compression techniques, it is conceivable to eliminate some of the redundant information confined in images. Image compression diminishes the size in bytes of a graphics file without corrupting the quality of the image to an intolerable level. The lessening in file size permits more images to be deposited in a certain amount of disk.

Correspondence to: jayakrishna.s2007@gmail.com

Recommended for acceptance by João Manuel Tavares

https://doi.org/10.5565/rev/elcvia.1094

ELCVIA ISSN: 1577-5097

Published by Computer Vision Center / Universitat Autonoma de Barcelona, Barcelona, Spain 
It also lessens the time required for images to be sent over the Internet or downloaded from web. The system of image compression is not new at all. The innovation of Discrete Cosine Transform (DCT) in 1974 is really an important achievement for those who work on image compression [2]. The DCT can be considered as a discrete time version of the Fourier Cosine series. It is a close relative of Discrete Fourier Transform, a system for transforming a signal into fundamental frequency components.

Thus, DCT can be calculated with a Fast Fourier Transform like algorithm of complexity $O\left(n \log _{2} n\right)$. Unlike DFT, DCT is real valued and offers a better estimate of a signal with less coefficients. There are a number of means in which images can be compressed. There are two common compressed image formats namely Joint Photographic Experts Group (JPEG) [3] and Graphic Interchange Format (GIF) for the use in the Internet. The JPEG method established by ISO (International Standards Organization) and IEC (International Electro-technical Commission) is more commonly used for photographs, while the GIF method is commonly used for line art and other images in which geometric shapes are quite simple.

In 1992, JPEG established the first international standard for still image compression where the encoders and decoders are based on DCT. The JPEG standard specifies three modes namely sequential, progressive, and hierarchical for lossy coding, and one mode for lossless coding. The concert of the coders of JPEG commonly cut down at low bit-rates mainly because of the underlying block-based DCT [4]. The baseline JPEG coder is the sequential encoding in its simplest form [5]. More recently, the wavelet transform has emerged as a cutting edge technology, in the arena of image analysis. Wavelets are a mathematical tool for hierarchically decomposing functions. Although rooted in approximation theory, signal processing, and physics, wavelets have also been applied to many difficulties in Computer Graphics including image editing and compression, automatic level-of detail control for editing and rendering curves and surfaces, surface reconstruction from contours and fast methods for solving simulation problems in 3D modeling, global illumination, and animation [6]. Wavelet-based coding offers significant progresses in picture superiority at higher compression ratios [7]. Over the past few years, a variety of commanding and sophisticated waveletbased schemes for image compression have been developed and realized.

The performance of wavelet based image compression standards completely lie on the wavelet domain of the input image. Though the basis function is not a sinusoidal signal, the basis signal will be some other signal supposed to be the same for any application and/or any input image. Out of some standard wavelets at a time one wavelet is arbitrarily chosen for a specific application. This may not yield good results for all the problem at hand. The wavelet basis function should be chosen based on the problem at hand. Hence a methodology has to be framed to find out the wavelet function for a specific application. Towards this end a number of works are proposed in the literature including [8]-[11]. In a specific application, for example, image compression the input images can be of different kind, hence depending on the input signal the wavelet bases have to be adapted to the input signal. Towards this end as well, a number of works are proposed [12]-[16]. In this paper review of wavelet based image compression, review of optimum wavelet selection, commonly used basis functions, factors to consider in choosing the basis functions are presented.

\section{Wavelet-based Image Compression}

\section{a. Fundamentals of Image Compression}

There are two types of image compression; lossy and lossless. With lossless compression, the original image is recovered exactly after decompression. Unfortunately, with images of natural scenes it is rarely possible to obtain error-free compression at a rate beyond 2:1. Much higher compression ratios can be obtained if some error, which is usually difficult to perceive, is allowed between the decompressed image and the original image. This is lossy compression. In many cases, it is not necessary or even desirable that there be error-free reproduction of the original image. For example, if some noise is present, then the error due to that noise will usually be significantly reduced via some denoising method. In such a case, the small amount of error introduced by lossy compression may be acceptable. Another application where lossy compression is acceptable is in fast transmission of still images over the Internet. The paper concentrates on the design of better wavelets for image compression, hence though there exists a wide variety of compression techniques; here, only wavelet based image compression standards are considered. The methods of compression that are considered are: the Embedded Zerotree Wavelet (EZW) algorithm, the Set Partitioning in Hierarchical Tree (SPIHT) algorithm, the Spatial-oriented Tree Wavelet (STW) algorithm, the Wavelet 
Difference Reduction (WDR) algorithm, and the Adaptively Scanned Wavelet Difference Reduction (ASWDR) algorithm. These are relatively recent algorithms which achieve some of the lowest errors per compression rate and highest perceptual quality yet reported. Before examining these algorithms, we shall outline the basic steps that are common to all wavelet-based image compression algorithms. The five stages of compression and decompression are shown in Fig.1 and 2. All of the steps shown in the compression diagram are invertible, hence lossless, except for the Quantize step. Quantizing refers to a reduction of the precision of the floating point values of the wavelet transform, which are typically either 32-bit or 64-bit floating point numbers. This leads to rounding error. These approximate, quantized, wavelet transforms will produce approximations to the images when an inverse transform is performed thus creating the error inherent in lossy compression.

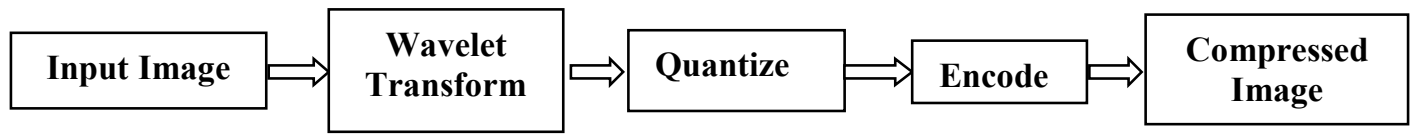

Fig. 1 Compression of an image

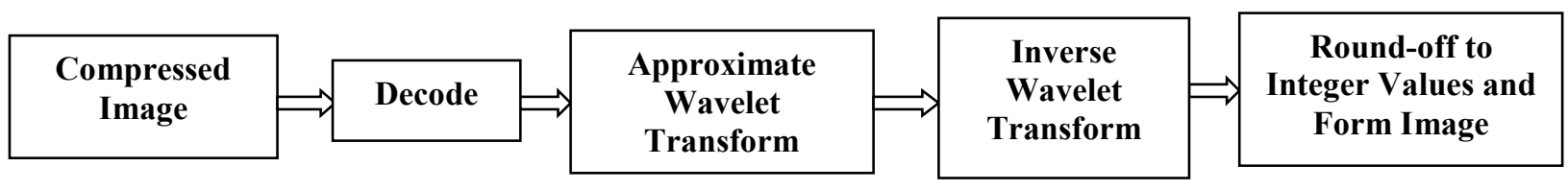

Fig. 2 Decompression of an image

The relationship between the Quantize and the Encode steps is the crucial aspect of wavelet transform compression. Each of the algorithms described below takes a different approach to this relationship. The purpose served by the Wavelet Transform is that it produces a large number of values having zeroed or near zeroed, magnitudes.

\section{b. Methods of Image Compression}

The EZW coder was introduced by Shapiro [17]. It is a quantization and coding strategy that incorporates some characteristics of the wavelet decomposition. Just as the quantization and coding approach used in JPEG standard [18], which was motivated by the characteristics of the coefficients, were superior to the generic zonal coding algorithms, the EZW approach and its descendants significantly outperform some of the generic approaches. The particular characteristic possessed by the EZW algorithm is that there are wavelet coefficients in different sub bands that represent the same spatial location in the image. If the decomposition is such that the size of the different sub bands is different, then a single coefficient in the smaller sub band may represent the same spatial location as multiple coefficients in the other sub bands. Given a threshold $\mathrm{T}$, if a given coefficient has a magnitude greater than $\mathrm{T}$, it is called a significant coefficient. If the magnitude of the coefficient is less than $\mathrm{T}$, it is insignificant and all its descendants have magnitudes less than $\mathrm{T}$, then the coefficients is called a zero tree root. If the coefficient is insignificant but some of its descendants have a value greater than $\mathrm{T}$, then the coefficient is called an isolated zero. The EZW algorithm is a multi-pass algorithm, with each pass consisting of two steps: significant map encoding or the dominant pass, and refinement or the subordinate pass.

The SPIHT algorithm is a generalization of the EZW algorithm and was proposed by Amir Said and William Pearlman [19]. Recall that in EZW we transmit a lot of information for little cost when we declare an entire sub tree to be insignificant and represent all the coefficients in it with a zerotree root label $z r$. The SPIHT algorithm uses a partitioning of the trees in a manner that tends to keep insignificant coefficients together in larger subsets. The partitioning decisions are binary that are transmitted to the decoder, providing a significance map encoding that is more efficient than EZW.

In fact, the efficiency of the significance map encoding in SPIHT is such that arithmetic coding of binary decisions provides very little gain. The thresholds used for checking significance are powers of two, so in essence the SPIHT algorithm sends the binary representation of the integer value of the wavelet coefficients. As in EZW, the significance map encoding, or the set partitioning and ordering step, is followed by a refinement step in which the representations of the significant coefficients are refined. The data structure used by the SPIHT algorithm is similar to that used by the EZW algorithm - although not the same. The wavelet coefficients are again divided into trees originating from the lowest resolution band. 
The coefficients are grouped into $2 \times 2$ arrays that, except for the coefficients in band I, are offspring of a coefficient of a lower resolution band. The coefficients in the lowest resolution band are also divided into $2 \times 2$ arrays. However, unlike the EZW case, all but one of them are root nodes. The coefficient in the top-left corner of the array does not have any offspring. The Fig. 3 shows the data structure for seven-band decomposition.

The trees are further partitioned into four types of sets, which are sets of coordinates of the coefficients:

- $O(I, j)$ : This is the set of coordinates of the offspring of the wavelet coefficient at location $(i, j)$. As each node can either have four offspring or non, the size of $\mathrm{O}(\mathrm{i}, \mathrm{j})$ is either zero or four. For example, in Fig. 3 the set $O(0,1)$ consists of the coordinates of the coefficients $b_{1}, b_{2}, b_{3}$ and $b_{4}$.

- $\mathrm{D}(\mathrm{i}, \mathrm{j})$ : This is the set of all descendants of the coefficient at location $(\mathrm{i}, \mathrm{j})$. Descendants include the offspring, the offspring of the offspring, and so on. For example, in Fig. 3, the set $\mathrm{D}(0,1)$ consists of the coordinates of the coefficients $b_{1}, \ldots, b_{4}, b_{11}, \ldots, b_{14}, \ldots, b_{44}$. Because the number of offspring can either be zero or four, the size of $\mathrm{D}(\mathrm{i}, \mathrm{j})$ is either zero or a sum of powers of four.

- $\mathrm{H}$ : This is the set of all root nodes - essentially band I in the case of Fig. 3.

- $\mathrm{L}(\mathrm{i}, \mathrm{j})$ : This is the set of coordinates of all the descendants of the coefficient at location $(\mathrm{i}, \mathrm{j})$ except for the immediate offspring of the coefficient at location (i,j).

A set $\mathrm{D}(\mathrm{i}, \mathrm{j})$ or $\mathrm{L}(\mathrm{i}, \mathrm{j})$ is said to be significant if any coefficient in the set has a magnitude greater than the threshold. Finally, thresholds used for checking significance are powers of two, so in essence the SPIHT algorithm sends the binary representation of the integer value of the wavelet coefficients. The bits are numbered with the least significant bit being the zeroth bit, the next bit being the first significant bit, and the $k$ th bit being referred to as the k-1 most significant bit. The algorithm makes use of three lists: the list of insignificant pixels (LIP), the list of significant pixels (LSP), and the list of insignificant sets (LIS). The LSP and LIS lists will contain the coordinates of coefficients, while the LIS will contain the coordinates of the roots of sets of type $\mathrm{D}$ or $\mathrm{L}$.

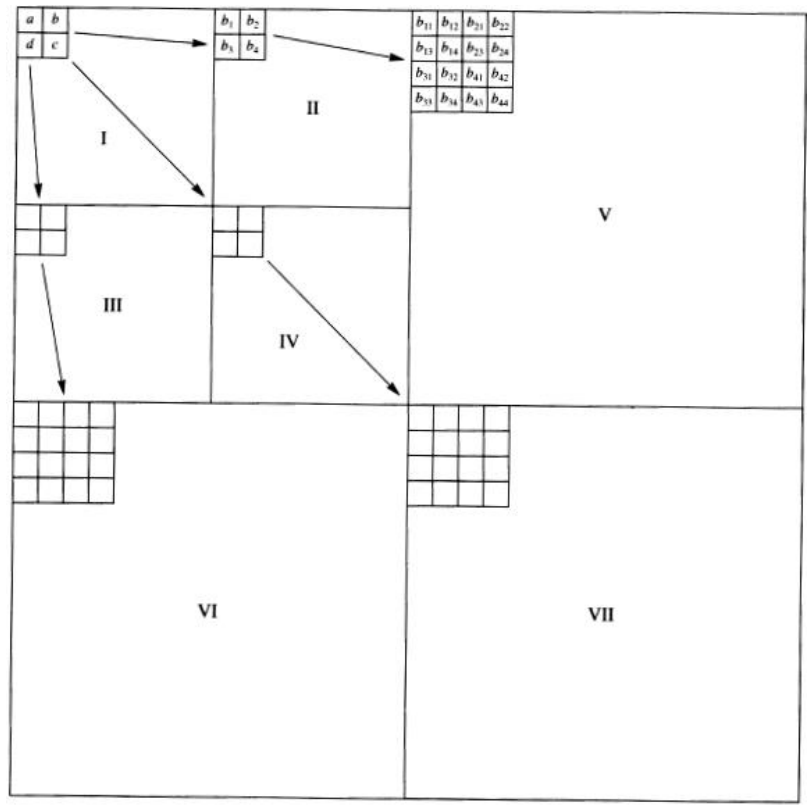

Fig. 3 Data Structure used in SPIHT coding

STW employs a various approach in coding the information of zero trees [20]. It is additional open eyed in its group of coding outputs than the EZW and SPIHT algorithm. EZW has the root location is marked by encoding only one symbol for the output R or I. In EZW consequently, the zerotrees offer slender descriptions of the locations of irrelevant values. The use of a state transition model is the various approach utilized in STW. From one threshold to consequent the locations of transform values undertake state transitions. So the number of bits required for encoding is thus condensed in STW with this design of state transitions. State transition model uses states IR, IV, SR and SV to score the locations rather than code for the outputs $\mathrm{R}$ and $\mathrm{I}$. Hence the states concerned are outline once knowing the import function $\mathrm{S}(\mathrm{m})$ and the descendent indices $\mathrm{D}(\mathrm{m})$. 
WDR technique combines run-length coding of the significance map with a well-organized illustration of the run-length symbols to produce relates embedded image coder [21]. SPIHT and WDR both have techniques, the zerotree data structure is precluded, but the embedding principles of lossless bit plane coding and set partitioning are sealed. Rather than using the zero trees, each coefficient during a off wavelet pyramid is appointed a linear position index in the WDR algorithm. Output of the WDR encoding can be arithmetically compressed. One of the defects of SPIHT is that it only implicitly locates the position of significant coefficients. This makes it difficult to perform operations, such as region selection on compressed data, which depend on the exact position of significant transform values. By region selection, also known as region of interest (ROI), we mean selecting a portion of a compressed image which requires increased resolution. This can occur, for example, with a portion of a low resolution medical image that has been sent at a low bpp rate in order to arrive quickly.

ASWDR is one of the leading enhanced image compression algorithms proposed by Walker [22]. This algorithm aims to get better the subjective perceptual qualities of compressed images and improve the results of objective alteration measures. ASWDR algorithm is a simple adaptation of the Wavelet Difference Reduction (WDR) algorithm. WDR algorithm employs a hard and fast ordering of the positions of wavelet coefficients, so ASWDR method employs a various order that aims to adapt itself to specific image features. The ASWDR adjusts the scanning order therefore as to predict locations of latest significant values. Scanning order of ASWDR dynamically adapts to the locations of edge details in an image, and this increases the declaration of these edges in ASWDR compressed images. Hence, ASWDR shows better perceptual qualities, especially at low bit rates, than WDR and SPIHT compressed images preserving all the features of WDR. One of the most recent image compression algorithms is the ASWDR algorithm. The adjective adaptively scanned refers to the fact that this algorithm modifies the scanning order used by WDR in order to achieve better performance. ASWDR adapts the scanning order so as to predict locations of new significant values. If a prediction is correct, then the output specifying that location will just be the sign of the new significant value the reduced binary expansion of the number of steps will be empty. Therefore a good prediction scheme will significantly reduce the coding output of WDR.

c. Review of Image Compression Algorithms

In 1985, image data compression arrangement by means of discrete transform is proposed [23]. Exploiting a cosine transform in image data compression has several standard performance profits, ensuing in the ability to achieve large compression ratios with small quality cost. Similarly, integration of a model of the human visual system (HVS) into an image data compression or eminence valuation method instinctively should and has often demonstrated to increase the performance. Evidently it should verify highly useful to pool the image cosine transform with a graphical model. In past, joining these two has been stuck by an essential problem resultant from the sight modification that is essential for proper cosine transform usage. A novel logical solution to this tricky, compelling the form of a straightforward multiplicative weighting function, is established in this effort. This clarification is eagerly appropriate to image data compression and quality valuation in combination with a pictorial model and the image data cosine transform. In the growth, relevant features of a HVS model are discoursed, and a superior version of the MSE quality valuation measure is set which would raise this degree's usefulness.

Gregory K. Wallace, in [18] proposed the JPEG Still picture compression standard. The evolving Joint Photographic Expert Group - JPEG image data compression standard is not a remedy that will resolve the myriad disquiets which must be solved before images specifically digital images combined in different applications that will eventually profit from them. For instance, if two applications can't carry a conversation of uncompressed images since they may use different aspect ratios, mismatched colour spaces, dissimilar dimensions, etc. then a common compression scheme will not assist. Though, numerous applications are held as storage or transmission costs, because of the argument over which compression technique to utilize, or since VLSI codecs are costly due to low bulks. For this kind of applications, the systematic practical assessment, selection, testing, justification and documentation work which JPEG committee have accomplished is anticipated to return an accepted international standard which endure the tests of excellence and time. As various imaging and image processing applications turned out to be progressively applied on open networked computing organizations the decisive measure of the committees' success will be when JPEG compressed images come to be considered and even taken for approved as just one more data type, as text and graphics are in 1990's, which turned absolutely true nowadays. 
In 1999, Sergio D. Servetto presented an investigational study of the statistical stuffs of wavelet coefficients of image data, as well as the strategy of two different morphology based image coding procedures that make use of these indications [24]. A striking property of the suggested approaches is that, by a humble variation of quantizer, the same basic process profits high performance entrenched or immovable rate coders. Additional significant property is that the shape data of morphological sets used in this coder is coded indirectly by the values of wavelet coefficients; therefore evading the use of obvious and rate expensive shape descriptors. These systems, despite the fact attaining nearly the same objective performance of state of the art zero tree approaches, are able to yield reconstructions of a somewhat higher perceptual quality, because of a property of joint compression and noise reduction they exhibit.

In 2005, Bushra K. Al-Abudi and Loay A. George designated a colour image compression system based on Haar wavelet transform [25]. The vertical and horizontal Haar filters are composed to build four 2D filters, such filters smeared straightforward to the image data to speed up the execution of the Haar wavelet. Haar wavelet was used to map the image to frequency groups; each group was allotted a weighting aspect bestowing to its subjective importance. Such weighting issues were comprised over and done with the computation method of the amount of bits essential to present the quantized indices of the wavelet coefficients. Transmission progressive and Zero tree mechanism were considered and realized using the encoding and decoding method. The examination results have shown that the concert of the proposed technique is much superior, where the built images are less distorted and compressed with high factor.

In 2007, Kamrul Hasan Talukder and Koichi Harada presented a low complex two dimensional image data compression technique by wavelet transforms as the basis functions and the approach to measure the quality of the compressed image data [26]. The specific wavelet chosen and used here is the humblest wavelet form namely the Haar Wavelet. The 2D DWT has been applied and the detail matrices from the data matrix of the image have been estimated. The rebuilt image is manufactured by the expected detail matrices and data matrix provided by the wavelet. The quality of the compressed images has been assessed using some design metrics.

In 2008, V. Bruni, B. Piccoli and D. Vitulano proposed a wavelet based denoising model [27]. The model described the wavelet transform of a general signal as a superimposition of predefined basic atoms. In 2008, V. M. Mankar, T. S. Das, S. Sarkar and S. K. Sarkar analysed the performance of a blind watermarking scheme based on discrete wavelet frame as compared to the traditional orthogonal wavelet expansion [28]. The over complete representation offered by the redundant frame facilitates the identification of significant image features via a simple correlation operation across scales.

In 2010, Raja S.P, Suruliandi A presented a comparison of different compression schemes on wavelet decomposed image data [29]. The schemes involved in the comparison are SPIHT, SOFM and EZW. These methods are more effective and deliver an enhanced quality. These work emphases significant feature of wavelet in compression of still images, comprising the extent to which the quality of image is corrupted by the process of wavelet compression and de-compression. The above methods have been efficaciously utilized in many fields. The methods are compared using the design metrics MSE and PSNR.

In 2010, an effective and fast image compression system based on all level curvelet coefficients with SPIHT was proposed [30]. In 2010, a new lossless image compression technique is proposed in [31]. For continuous and discrete cases, wavelet transforms and wavelet packet transform (WPT) has arose as popular methods. Although integer wavelet utilizing the lifting scheme significantly decreases the computation time, the authors have proposed a totally new methodology for additional speeding up the computation of transform. First, wavelet packet transform and lifting schemes are presented.

Then an application of the lifting scheme to wavelet packet transform is presented which clues to the generation of integer wavelet packet transform (IWPT). The IWPT yields an illustration which can be lossless, as it maps integer valued series onto the integer valued coefficients. The idea of WPT is utilized to transform the still and colour images. Integer WPT tree can be constructed by repeating the single wavelet decomposition step on both the low pass and high pass twigs, with rounding off in order to attain the integer transforms. Therefore, the proposed method offers good compression ratio CR.

In 2011, a novel near lossless compression system is proposed in [32] and which yields considerably enhanced compression rates. In this method, base points, direction images and D-value images are acquired from RGB colour space image by conversion. Base points, direction images are determined by binary coding, dispersed arithmetic coding. Wavelet coefficients of D-value images are encoded by adaptive Huffman coding (AHC). 
As a consequence, high over all compression rates, superior diagnostic image quality and enhanced performance parameters are attained. The procedure is verified on investigational medical images from diverse modalities and diverse body districts and results are described.

In 2011, an effective image coding process Fast SPIHT has been proposed in [33]. The process presents the lifting wavelet and a wavelet basis based on HVS, the classification of the List of Insignificant Sets (LIS) is removed in the new compression process, the hierarchical trees in wavelet decomposition are coded in the same way from son generation to grandson generation. The investigational outcome demonstrates that the reconstructed image of this algorithm has superior visual effect and furthermore increases coding speed.

In 2012, Leni, P.E, et al, present a new method for image data coding based on an exceptional representation of image data [34]. The proposed method is different from the most of the methods in the literature till that time because the compression is not straightaway accomplished on the image pixels, but is applied to a corresponding mono-variate representation of the wavelet domain of image data. More accurately, an adaptation of Kolmogorov superposition theorem proposed by Igelnik and known as the Kolmogorov spline network where the image is estimated by sums and compositions of specific monovariate functions was deliberated. By using this representation, the authors trade the local connectivity and the traditional line per line scanning, in exchange of a more adjustable and uni-variate representation of image data, which permits tackling the compression jobs in a primarily different representation. The contributions lie in the numerous policies offered to adapt the Kolmogorov spline network algorithm, including the mono-variate structure, numerous simplification policies, and the proposal of a more appropriate representation of the new image using wavelets and the incorporation of this system as an added layer in the JPEG 2000 compression machine, demonstrated for many images at diverse bit rates.

In 2012, Hong-jun Li. et al, use the character of wavelet coefficients, and apply the grey relational theory in coefficients relational narration, and then propose an image data compression scheme through grey relational theory [35]. The authors have classified the coefficients with respect to their characters in diverse domains and build the sparse representation technique under diverse types of coefficients. The algorithm decreases the computational difficulty and increases the ability of image sparse representation. It attains an effective way of image data compression. The simulation results demonstrate that the proposed compression process based on grey relational theory is better than the other procedures both in the pictorial quality and PSNR.

In 2013, Jaya Krishna Sunkara, E Navaneethasagari et al. presented a video compression algorithm using wavelet transform [36]. The method involves conversion of 3D signal into $2 \mathrm{D}$ signal using accordion representation. Then, a wavelet based compression technique is utilized. In 2013, easy path wavelets transform (EPWT), a wavelet domain image data compression standard that means in adaptive vicinities of image intensity points, is used in [37], for the sparse representation of 2D data. In [38], an image compression standard based on DWT along with a security tool was proposed. A probabilistic triangular shuffling scheme was sued to improve the security of the image. The proposed algorithm tends to achieve $67-70 \%$ compression ratio wit high security. Probability of security breach was utilized as a measure of image security.

In 2015, iris image compression using wavelet transformed image was considered in [39]. The spatial oriented tree wavelet coding techniques such as EZW and SPIHT are used. The basic Haar wavelet was used in this work. PSNR, MSE, and CRs are calculated. In 2016, probability distribution estimation was proposed which is supposed to be used for autoregressive pixel predictive image coding [40]. This work is focused on mean intensity prediction of the pixel to be transmitted.

Miguel Hernandez-Cabronero et al. proposed compression of DNA microarray images [41][42]. First, the consistent structure of the Relative Quantizer intervals is oppressed to define a lossy-to-lossless coding algorithm referred as Progressive Relative Quantizer (PRQ) coder. Second, a superior version that prioritizes a region of interest, called the PRQ-region of interest (ROI) coder, is described.

In 2016, Karthik. R and Menaka. R analysed the characteristics of ischemic stroke injuries using wavelets [43]. Four different wavelet functions namely Daubechies, Symlet, Coiflet and De-Meyer were applied to the different datasets and the resulting observations were examined based on their feature statistics. The characteristics of normal brain tissues and abnormal lesion structures using a 3-level wavelet decomposition. In 2019, Chuxi Yang, Yan Zhao and Shigang Wang proposed to use deep neural networks for image compression in the wavelet transform domain [44]. The wavelet decomposition of digital image was done. But only the low frequency band is cried to the receiver yielding higher compression ratio. 
At the decompression, the three high frequency bands were estimated using the low frequency band. Many compression variants were proposed in the literature based on wavelets [45][46].

Transform based image compression schemes, first involve the transformation of spatial information in to another domain. For example, the DCT transforms an image into the frequency domain. The goal of the transformation is a compact, complete representation of the image. The transform should decorrelate the spatially distributed energy into fewer data samples such that no information is lost. Orthogonal transforms have the feature of eliminating redundancy in the transformed image. Compression take place in the second step when the transformed image is quantized. The inverse transform reconstructs the compressed image in the spatial domain. Since the quantization process is not invertible, the reconstruction cannot perfectly recreate the original image. This type of compression is called lossy. In transform based image compression, entropy coding normally follows the quantization stage.

Entropy coding diminishes the redundancy in the bit stream and is fully invertible at the decoding end. So, it is lossless and usually gives about 0.4-0.6 dB gain in the PSNR. As previously mentioned in waveletbased image coding, the coding performance depends on the choice of wavelets. Several wavelets which provide suboptimal coding performance have been proposed in the literature. Recently, a few approaches for selecting the optimal filter bank in an image coder have been proposed in the literature [47][48]. Generally, a wavelet providing optimal performance for the whole image is selected. However, a few cases have been reported where spatially adapted filter banks were employed.

We note that finding the optimal wavelet for a particular image is a computationally intensive task. Tewfik, et al. [48] have proposed a technique to find the best wavelet basis by maximizing the $L^{2}$-norm of the wavelet approximated signal. Caglar, et al. [47] have proposed techniques for designing wavelets which are optimal in the statistical sense. The complexity of these algorithms increases considerably with the filter order. The performance of wavelet-based coding also depends on the wavelet decomposition structure. Ramchandran, et al. [49] have proposed a technique, based on Lagrangian optimization, to find the best basis subtree. This technique minimizes the global distortion for a given bit-rate. However, this algorithm is computationally expensive. Before employing a computationally intensive procedure for finding the optimal basis, it will be helpful to know, a priori, if appreciable gain can be achieved by using the optimal basis over known "good" wavelets.

\section{Example Scaling and Wavelet Functions}

In this section different wavelet functions routinely used in image processing are revealed. The effect of the basis function on the performance of a particular task depends on various properties of the basis functions. The properties include vanishing moments, symmetry, compact support and etc.

\section{a. Piecewise Constant}

The simplest example of scaling function is the Haar scaling function given in equation (1).

$$
\phi(t)=\left\{\begin{array}{cc}
1 & 0 \leq t \leq 1 \\
0 & \text { elsewhere }
\end{array}\right.
$$

The scaling functions satisfies the scaling equation given in equation (2).

$$
\phi(t)=\phi(2 t)+\phi(2 t-1) .
$$

Most of the times, the coefficients $\mathrm{p}_{\mathrm{j}}$ vanish outside the range $0 \leq j \leq J$. Hence, the generalized scaling equation becomes

$$
\phi(t)=\sum_{j=0}^{J} p_{j} \phi(2 t-j)
$$

and its symbol polynomial is given in equation (4).

$$
P(z)=\frac{1}{2} \sum_{j=0}^{J} p_{j} z^{j}
$$

The value of the scaling function at the integers satisfy the infinite system of linear equations given in equation (5). 


$$
\phi(i)=\sum_{j \in Z} p_{j} \phi(2 i-j)=\sum_{j \in Z} p_{2 i-j} \phi(j)
$$

When $\phi$ has the compact support [0, J], it reduces to finite system and is given in equation (6).

$$
\left(\begin{array}{c}
\phi(0) \\
\phi(1) \\
\phi(2) \\
\phi(3) \\
\phi(4) \\
\vdots \\
\phi(J)
\end{array}\right)=\left(\begin{array}{lllllllll}
p_{0} & & & & & & & \\
p_{2} & p_{1} & p_{0} & & & & \\
p_{4} & p_{3} & p_{2} & p_{1} & p_{0} & & \\
p_{6} & p_{5} & p_{4} & p_{3} & p_{2} & \ddots & & \\
\ddots & \ddots & \ddots & p_{5} & p_{4} & \ddots & & \\
& & & & \ddots & \ddots & \ddots & \ddots & \ddots \\
& & & & & & & p_{J}
\end{array}\right)\left(\begin{array}{c}
\phi(0) \\
\phi(1) \\
\phi(2) \\
\phi(3) \\
\phi(4) \\
\vdots \\
\phi(J)
\end{array}\right)
$$

In the case of Haar scaling function $\mathrm{p}_{0}=\mathrm{p}_{1}=1$ and

$$
P(z)=\frac{1+z}{2}
$$

Its Fourier transform is

$$
\hat{\phi}(w)=\frac{1-e^{-i w}}{i w}
$$

The scaling and wavelet functions are shown in the Fig. 4.

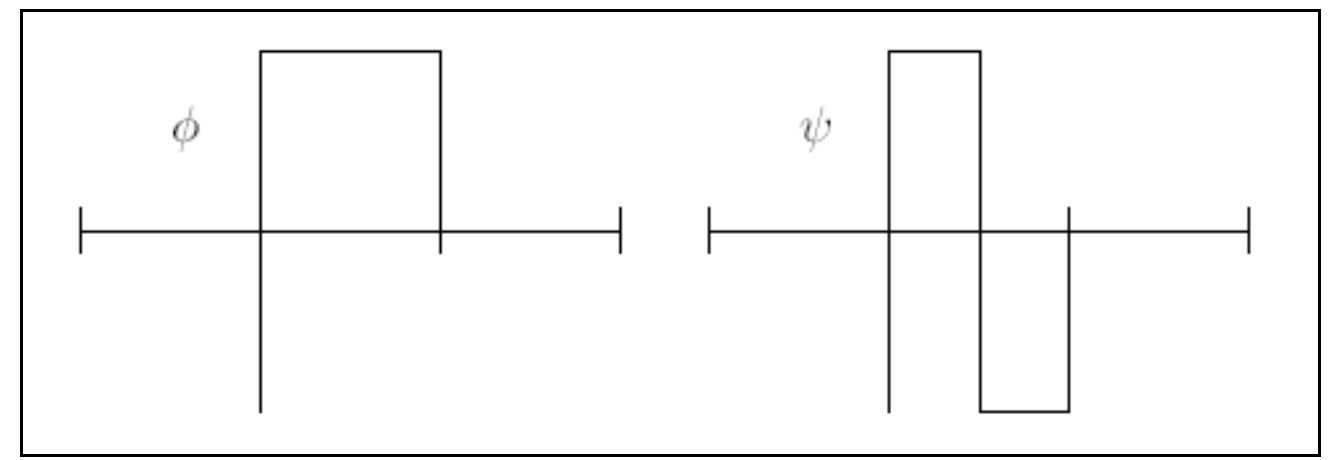

Fig. 4 Piecewise constant scaling and wavelet functions

$\mathrm{V}_{0}$ is then the space of piecewise - constant functions with discontinuous at the integers, which reproduces all constants. $\mathrm{P}$ is the $2 \times 2$ unit matrix.

$$
P=\left(\begin{array}{ll}
1 & 0 \\
0 & 1
\end{array}\right)
$$

whose repeated Eigenvalue of 1 reflects the fact that $\phi(0)$ and $\phi(1)$ are indeterminate. The Eigenvalues are \pm 1 .

\section{b. Piecewise Linear}

A more interesting example of a scaling function is the hat function given in equation (10).

$$
\phi(t)=\left\{\begin{array}{lr}
t & (0 \leq t \leq 1) \\
2-t & (1 \leq t \leq 2) \\
0 & (\text { Otherwise }))
\end{array}\right.
$$

satisfying the scaling equation

$$
\phi(t)=\frac{1}{2} \phi(2 t)+\phi(2 t-1)+\frac{1}{2} \phi(2 t-2)
$$

So that $\mathrm{p}_{0}=\mathrm{p}_{2}=1 / 2, \mathrm{p}_{1}=1$ and

$$
P(z)=\left(\frac{1+z}{2}\right)^{2}
$$

Its Fourier transform is 
$\hat{\phi}(w)=\left(\frac{1-e^{-i w}}{i w}\right)^{2}$

The scaling and wavelet functions are shown in the Fig. 5.

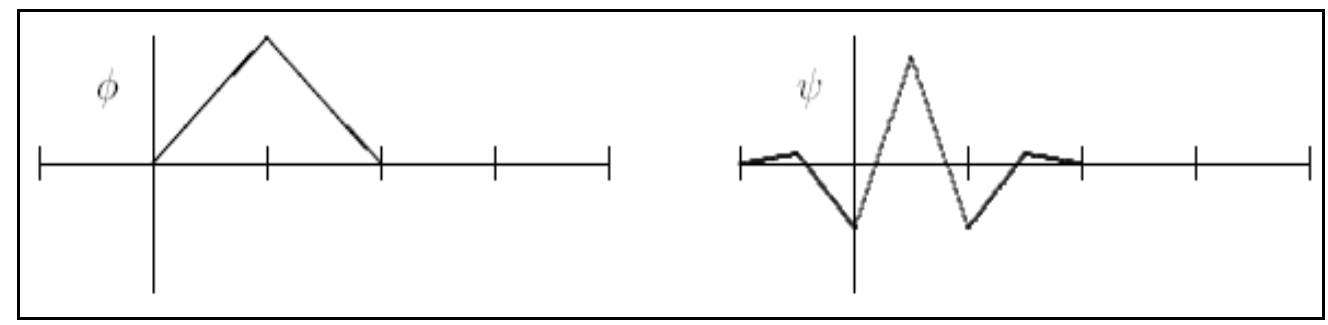

Fig. 5 Piecewise linear scaling and wavelet functions

\section{c. Biorthogonal Scaling Functions and Wavelets}

It is somewhat inconvenient that if $\phi$ and $\psi$ have compact support then their duals $\tilde{\phi}$ and $\tilde{\psi}$ must have infinite support. It is often possible to avoid this difficulty by going over to biorthogonal multiresolution [50]. Starting with the nested sequence of subspaces

$\ldots \subset V_{-1} \subset V_{0} \subset V_{1} \subset V_{2} \subset \cdots L_{2}(R)$

and their associated scaling function $\phi$, let us define the sequences of subspaces

$$
\begin{aligned}
& \cdots, W_{-1} \subset V_{0}, W_{0} \subset V_{1}, W_{1} \subset V_{2}, \cdots \\
& \cdots \subset \tilde{V}_{-1} \subset \tilde{V}_{0} \subset \tilde{V}_{1} \subset \tilde{V}_{2} \subset \cdots L_{2}(R) \\
& \cdots, \tilde{W}_{-1} \subset \tilde{V}_{0}, \tilde{W}_{0} \subset \tilde{V}_{1}, \tilde{W}_{1} \subset \tilde{V}_{2}, \cdots
\end{aligned}
$$

each having the translation and scaling properties and with $V_{K+1}=V_{K} \oplus W_{K}$ and $\tilde{V}_{K+1}=\tilde{V}_{K} \oplus \tilde{W}_{K}$. However, these two decompositions are now to be not orthogonal i.e. $\left(V_{K} \perp W_{K}\right)$ and $\left(\widetilde{V}_{K} \perp \widetilde{W}_{K}\right)$ but biorthogonal, in the sense that

$$
V_{K} \perp \tilde{W}_{K}, \tilde{V}_{K} \perp W_{K}
$$

More precisely,

$$
\begin{aligned}
& \int_{R} \phi(s) \widetilde{\phi}(s-j) d s=\int_{R} \psi(s) \widetilde{\psi}(s-j) d s=\delta_{j 0} \forall j \in Z, \\
& \int_{R} \phi(s) \widetilde{\psi}(s-j) d s=\int_{R} \psi(s) \widetilde{\phi}(s-j) d s=0 \quad \forall j \in Z .
\end{aligned}
$$

As well as the scaling equations for $\phi$ and $\psi$ with symbols $\mathrm{P}(\mathrm{z})$ and $\mathrm{Q}(\mathrm{z})$, here will again be further scaling equations with symbols $\tilde{P}(z)$ and $\tilde{Q}(z)$, and the biorthogonal conditions will lead to relationships

$$
\begin{aligned}
& \tilde{P}(z) P(1 / z)+\tilde{P}(-z) P(-1 / z)=1, \\
& \tilde{P}(z) Q(1 / z)+\tilde{P}(-z) Q(-1 / z)=0, \\
& \tilde{Q}(z) P(1 / z)+\tilde{Q}(-z) P(-1 / z)=0, \\
& \tilde{Q}(z) Q(1 / z)+\tilde{Q}(-z) Q(-1 / z)=1,
\end{aligned}
$$

Given any $\mathrm{P}$ such that $\mathrm{P}(1)=1, \mathrm{P}(-1)=0$, then there may be many possible $\tilde{P}$ such that $\widetilde{P}(1)=1$. Taking any such $\tilde{P}$ and if one let

$$
\begin{aligned}
& Q(z)=-z \tilde{P}(-1 / z), \\
& \tilde{Q}(z)=-z p(-1 / z) .
\end{aligned}
$$

then it is easy to see that all the four equations will hold. For example, consider the piecewise linear (Hat) scaling function with the following symbol

$$
P(z)=\left(\frac{1+z}{2}\right)^{2}=\frac{1+2 z+z^{2}}{4}
$$


Then to satisfy the above equations one way is to take

$$
\tilde{P}(z)=\frac{-1 / z+2+6 z+2 z^{2}-z^{3}}{8}=\left(\frac{1+z}{2}\right)^{2} \frac{-1 / z+4-z}{2}
$$

Then

$$
Q(z)=\frac{-1 / z^{2}-2 / z+6-2 z-z^{2}}{8}, \tilde{Q}(z)=\frac{-1 / z+2-z}{4} .
$$

The corresponding scaling and wavelet functions are shown in Fig. 6.

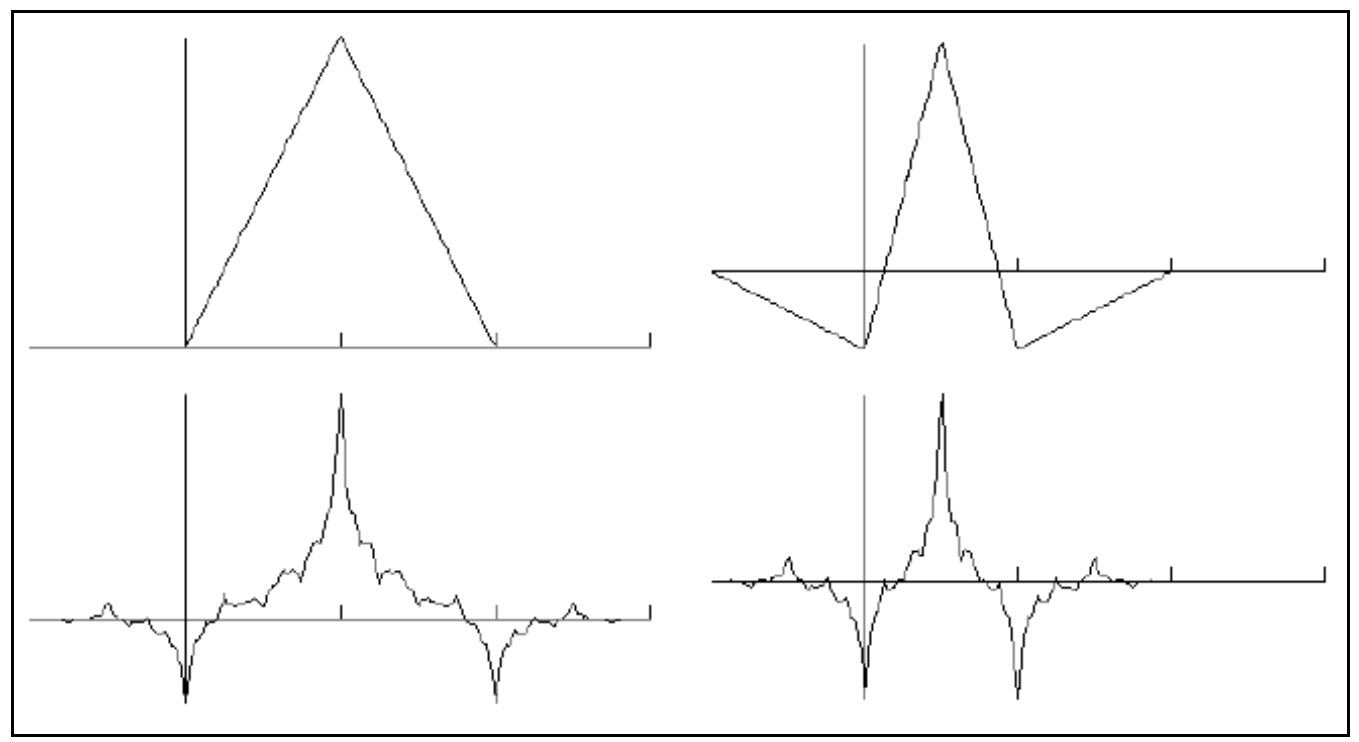

Fig. 6 Biorthogonal scaling and wavelet functions

\section{$4 \quad$ Selection of Wavelet Bases}

The most important aspect of wavelet analysis is the possibility to construct wavelet bases of $\mathrm{L}_{2}$ [51][52]. The wavelet basis is generally given by the set of detailed and translated versions of the mother wavelet. Hence it is possible to represent a signal through its wavelet expression

$$
f=\sum_{i} \sum_{k \in Z} c_{i, k} \psi_{i, k}
$$

where the wavelet coefficients $\mathrm{c}_{\mathrm{i}, \mathrm{k}}$ are obtained through the inner product $c_{i, k}=\left\langle f, \bar{\psi}_{i, k}\right\rangle$

The function $\bar{\psi}$ is the dual analysis wavelet; in the orthogonal case $\psi$ and $\bar{\psi}$ are identical. The important point is that, in the discrete case, the above decomposition provides a one-to-one representation of the signal in terms of its wavelet coefficients. Data compression as well as noise reduction can be achieved by quantization in wavelet domain or by simply discarding certain coefficients that are insignificant.

This form of orthogonal wavelet decomposition was found to be very useful for image coding [53]. There have also been specific applications of wavelet compression to medical images, including MR images, digital mammograms, as well as 3D data sets. Most applications of wavelet bases exploit their ability to efficiently approximate particular classes of functions with few non-zero wavelet coefficients. This is true not only for data compression but also for noise removal and fast calculations. The design of $\psi$ must therefore be optimized to produce a maximum number of wavelet coefficients $\left\langle f, \psi_{j, n}\right\rangle$ that are close to zero. A function $\mathrm{f}$ has few non-negligible wavelet coefficients if most of the fine-scale (high-resolution) wavelet coefficients are small. This depends mostly on the regularity of $f$, the number of vanishing moments of $\psi$ and the size of its support. To construct an appropriate wavelet from a conjugate mirror filter $\mathrm{h}[\mathrm{n}]$, we relate these properties to conditions on $\hat{h}(w)$. 


\section{a. Vanishing Moments}

The wavelet function $\psi$ has $\mathrm{p}$ vanishing moments if

$$
\int t^{k} \psi(t) d t=0 \quad \text { for } 0 \leq k<p
$$

This means that $\psi$ is orthogonal to any polynomial of degree $\mathrm{p}-1$. If $f$ is regular and $\psi$ has enough vanishing moments then the wavelet coefficients $\left|\left\langle f, \psi_{j, n}\right\rangle\right|$ are small at fine scales $2^{\mathrm{j}}$.

Indeed, if $\mathrm{f}$ is locally $\mathrm{C}^{\mathrm{k}}$, then over a small interval it is well approximated by a Taylor polynomial of degree $\mathrm{k}$. If $\mathrm{k}<\mathrm{p}$, then wavelets are orthogonal to this Taylor polynomial and thus produce small amplitude coefficients at fine scales.

\section{b. Size of Support}

If $\mathrm{f}$ has an isolated singularity at $\mathrm{t}_{0}$ and if $\mathrm{t}_{0}$ is inside the support of $\psi_{j, n}(t)=2^{-j / 2} \psi\left(2^{-j} t-n\right)$ then $\left\langle f, \psi_{j, n}\right\rangle$ may have a large amplitude. If $\psi$ has a compact support of size $\mathrm{K}$, at each scale $2^{\mathrm{j}}$ there are $\mathrm{K}$ wavelets $\psi_{j, n}$ whose support includes $\mathrm{t}_{0}$. To minimize the number of high amplitude coefficients we must reduce the support size of $\psi$. The following theorem relates the support size of $\mathrm{h}$ to the support of $\phi$ and $\psi$.The scaling function $\&$ has a compact support if and only if $\mathrm{h}$ has a compact support and their support are equal. If the support of $\mathrm{h}$ and $\phi$ is $[\mathrm{N} 1, \mathrm{~N} 2]$ then the support of $\psi$ is $[(\mathrm{N} 1-\mathrm{N} 2+1) / 2,(\mathrm{~N} 2-\mathrm{N} 1+1) / 2]$.

\section{c. Support versus Moments}

The support size of a function and the number of vanishing moments are a priori independent. The constraints imposed on orthogonal wavelets imply that if $\psi$ has $\mathrm{p}$ vanishing moments then its support is at least of size $2 p-1$. Daubechies wavelets are optimal in the sense that they have a minimum size support for a given number of vanishing moments. When choosing a particular wavelet, we thus face a trade-off between the number of vanishing moments and the support size. If $\mathrm{f}$ has few isolated singularities and is very regular between singularities, we must choose a wavelet with many vanishing moments to produce a large number of small wavelet coefficients $\left\langle f, \psi_{j, n}\right\rangle$. If the density of singularities increases, it might be better to decrease the size of its support at the cost of reducing the number of vanishing moments. Indeed, wavelets that overlap the singularities create high amplitude coefficients. The multiwavelet construction of Geronimo, Hardin and Massupust offers more design flexibility by introducing several scaling functions and wavelets. However, multiwavelet decompositions are implemented with a slightly more complicated filter bank algorithm than a standard orthogonal wavelet transform.

\section{d. Regularity}

The regularity of $\psi$ has mostly a cosmetic influence on the error introduced by thresholding or quantizing the wavelet coefficients. When reconstructing a signal from its wavelet coefficients

$f=\sum_{j=-\infty}^{+\infty} \sum_{n=-\infty}^{+\infty}\left\langle f, \psi_{j, n}\right\rangle \psi_{j, n}$,

an error $\varepsilon$ added to a coefficient $\left\langle f, \psi_{j, n}\right\rangle$ will add the wavelet component $\varepsilon \psi_{j, n}$ to the reconstructed signal. If $\psi$ is smooth, then $\varepsilon \psi_{j, n}$ is a smooth error. For image coding applications, a smooth error is often less visible than an irregular error, even though they have the same energy. Better quality images are obtained with wavelets that are continuously differentiable than with the discontinuous Haar wavelet.

In 1994, Ronald A. DeVore proposed adaptive bases for image compression [54]. The analysis of performance of compression algorithm was based on the number of coefficients that need to be retained to achieve a given error tolerance. It was identified that the number of coefficients is not a completely fair assessment. Also it is stated that what really count is the number of bits that must be transmitted in order to reconstruct the compressed image. New problems arise when applying the adaptive wavelet basis algorithms. In this case the total number of potential coefficients is $4 \mathrm{~N}$ rather than $\mathrm{N}$. Even though the number of 
nonzero entries will be less than in the fixed basis case reflecting better performance of adaptive basis selection, the encoded file may be larger.

In 1995, Yan Zhuang and John S. Baras studied the problem of choosing an image based optimal wavelet basis with compact support for image data compression and provided a general algorithm for computing the optimal wavelet basis [55]. The authors parameterized the mother wavelet and scaling function of the wavelet systems through a set of real coefficients of the relevant quadrature mirror filter banks. They also introduced the concept of decomposition entropy as an information measure to describe the distance between a given digital image and its projection into the subspace spanned by the wavelet basis. The optimal basis for the given image is obtained through minimizing this information measure. The resulting subspace is used for image analysis and synthesis. A gradient based optimization algorithm is developed for computing the image based optimal wavelet basis. Experiments show improved compression ratios due to the application of the optimal wavelet basis and demonstrate the potential applications of the methodology in image compression. This method is also useful for constructing efficient wavelet based image coding systems.

In 1996, Michael G. Strintzis proposed optimal biorthogonal wavelet bases for signal decomposition [56]. The selection of scaling functions for optimal signal representation by general multidimensional biorthogonal wavelet bases is investigated. Criterion for optimality is the minimization of the mean-square approximation error at each level of the decomposition. Conditions are given under which the approximation error of the decomposition approaches zero as the level increases. Given arbitrary synthesis filters, the optimal corresponding analysis filters are determined. Globally optimal families of filters are also found, and suboptimal linear and nonlinear-phase filters for the realization of the optimal scaling functions are explicitly determined.

In 2000, Mojsilovic, Popovic and Rackov proposed the selection of optimal wavelet basis for texture classification [57]. The scope of this work was to investigate whether the properties of decomposition filters play an important role in texture description, and which feature is dominant in the selection of an optimal filter bank. Classification experiments with 23 Brodatz textures were performed. The investigation shows that the selection of the decomposition filters has a significant influence on the result of texture characterization. Finally, the paper ranks 19 orthogonal and biorthogonal filters, and establishes the most relevant criteria for choice of decomposition filters in wavelet-based texture characterization algorithms.

In 2003, Rajpoot, Wilson, Meyer, Coifman proposed adaptive wavelet packet basis selection for zerotree image coding [58]. A general zerotree structure for arbitrary wavelet packet geometry in an image coding framework was presented. A fast basis selection algorithm which uses a Markov chain based cost estimate of encoding the image using this structure is developed. As a result, the adaptive wavelet zerotree image coder has a relatively low computational complexity, performs comparably to the state-of-the-art image coders, and is capable of progressively encoding images. In 2005, Mandal, Panchanathan and Aboulnasr elaborated on choice of wavelets for image compression [59]. The authors show that searching for optimal wavelet does not always offer a substantial improvement in coding performance over "good" standard wavelets.

Some guidelines for determining the need to search for the "optimal" wavelets based on the statistics of the image to be coded are proposed. In addition, an adaptive wavelet packet decomposition algorithm based on the local transform gain of each stage of the decomposition was proposed. The proposed algorithm provides a good coding performance at a substantially reduced complexity. In 2007, Kharate, Patil and Bhale compared the performance image coding scheme when different wavelet bases are used [60]. This paper compares compression performance of Daubechies, Biorthogonal, Coiflets and other wavelets for different frequency images. Based on the result, it is proposed that proper selection of mother wavelet on the basis of nature of images, improve the quality as well as compression ratio remarkably.

In 2015, Maria Rehman, Imran Touqir, Wajiha Batool presented the performance of several wavelet bases in SPIHT coding [61]. Two types of wavelet bases are tested for SPIHT algorithm i.e. orthogonal and biorthogonal wavelet bases. The results of using coefficients of these bases are compared on the basis of Compression Ratio and Peak Signal to Noise Ratio. The paper shows that the use of biorthogonal wavelets bases is better than orthogonal wavelet bases. Out of biorthogonal wavelets, bior4.4 shows good results in SPIHT coding.

In 2015, Noor Kamal, Mohd Ali, Ahmad, Shabiul Islam and Escudero proposed selection of mother wavelet functions for multi-channel EEG signal analysis during a working memory task [62]. A comparative study to select the efficient mother wavelet basis functions that optimally represent the signal characteristics of the electrical activity of the human brain during a working memory task recorded through electro- 
encephalography was performed. Nineteen EEG electrodes were placed on the scalp following the 10-20 system. These electrodes were then grouped into five recording regions corresponding to the scalp area of the cerebral cortex. Sixty-second WM task data were recorded from ten control subjects. Forty-five MWT basis functions from orthogonal families were investigated. These functions included Daubechies (db1-db20), Symlets (sym1-sym20), and Coiflets (coif1-coif5). Using ANOVA, authors determined the MWT basis functions with the most significant differences in the ability of the five scalp regions to maximize their crosscorrelation with the EEG signals. The best results were obtained using "sym9" across the five scalp regions. Therefore, the most compatible MWT with the EEG signals should be selected to achieve wavelet denoising, decomposition, reconstruction, and sub-band feature extraction.

In 2016, Girisha Garg proposed a signal invariant wavelet function selection algorithm [63]. The author addresses the problem of mother wavelet selection for wavelet signal processing in feature extraction and pattern recognition. The problem is formulated as an optimization criterion, where a wavelet library is defined using a set of parameters to find the best mother wavelet function. For estimating the fitness function, adopted to evaluate the performance of the wavelet function, analysis of variance is used. Genetic algorithm is exploited to optimize the determination of the best mother wavelet function. For experimental evaluation, solutions for best mother wavelet selection are evaluated on various biomedical signal classification problems, where the solutions of the proposed algorithm are assessed and compared with manual hit-and-trial methods. The results show that the solutions of automated mother wavelet selection algorithm are consistent with the manual selection of wavelet functions. The algorithm is found to be invariant to the type of signals used for classification.

In 2018, Jiajia Liu, W.H. Siew, John J. Soraghan and Euan A. Morris proposed a new wavelet selection scheme for partial discharge signal detection [64]. Correlation-based wavelet selection scheme and energybased wavelet selection scheme are applied to select an appropriate wavelet basis function. A novel wavelet selection scheme, wavelet entropy-based wavelet selection scheme is proposed to provide an alternative to the above schemes for partial discharge denoising. In 2019, Sushree Satvatee Swain and Dipti Patra considered 12 leads to view the cardiac condition from various angles in ECG signal for accurate detection of Myocardial Infarction [65]. Various wavelet basis functions, i.e. Haar, Daubechies, Symlet, Coiflet and biorthogonal basis filters of different order were investigated for selecting the most suitable one for the detection of Myocardial Infarction. Consider the following simulation results obtained using various wavelet functions along with SPIHT given in Table I.

TABLE I: Compression performance of SPIHT with different wavelets

\begin{tabular}{lcccccc}
\hline Images & \multicolumn{2}{c}{ Board } & \multicolumn{2}{c}{ Coins } & \multicolumn{2}{c}{ Aerial } \\
\hline & CR & PSNR (dB) & CR & PSNR (dB) & CR & PSNR (dB) \\
\hline Haar & 1.94 & 35.55 & 2.74 & 38.40 & 2.15 & 35.89 \\
\hline db5 & 1.74 & 35.20 & 2.50 & 38.35 & 2.01 & 36.06 \\
\hline db10 & 1.61 & 22.56 & 2.33 & 36.20 & 1.87 & 32.86 \\
\hline bior1.3 & 1.78 & 19.11 & 2.47 & 31.87 & 1.97 & 28.81 \\
\hline bior2.2 & 1.83 & 20.52 & 2.64 & 32.41 & 2.12 & 31.80 \\
\hline coif1 & 1.89 & 19.76 & 2.74 & 34.13 & 2.16 & 31.11 \\
\hline coif3 & 1.73 & 35.10 & 2.38 & 38.40 & 1.90 & 36.08 \\
\hline sym2 & 1.82 & 21.85 & 2.57 & 31.17 & 2.06 & 24.72 \\
\hline sym3 & 1.79 & 19.89 & 2.56 & 34.63 & 2.06 & 31.67 \\
\hline Dmey & 0.96 & 19.84 & 1.41 & 35.61 & 1.11 & 32.71
\end{tabular}

The table shows compression results of SPIHT coding technique with different wavelets on three different images. The first image is a circuit board where the adjacent pixel redundancy is limited to few pixels, the second image is coins image where the adjacent pixel redundancy is very high. The last image is an Aerial survey image. The adjacent pixel redundancy in this case is present diagonally. The following are observed from the table. The wavelet ' $\mathrm{db} 10$ ' has shown poor performance compared to that of ' $\mathrm{db} 5$ ' and 'haar'. The wavelet 'bior1.3' has shown poor performance compared to that of 'bior2.2'. In the case of 
Coiflets, the compression ratio is better in case of 'coifl', but PSNR is better with 'coif3'. In the case of Symlets the compression ratio is almost similar, but except for 'board' image the PSNR is better with 'sym3'.

\section{Discussion}

In the last section, simulation results of image compression with SPIHT coding are presented. In SPIHT coding, the wavelet coefficients are compared with a threshold to place the coefficients in a respective lists. If the coefficient is less than the threshold, it is treated as insignificant otherwise as significant. The coding will be done in iteration wise. In each iteration, a new threshold value will be evaluated. Hence, a coefficient which treated as insignificant may become significant in further iterations.

For instance, if $\mathrm{k}=4,5$ coefficients can be obtained. They are 1/8, 4/8, 6/8, 4/8 and 1/8. After normalizing, these values can be used as coefficients of one of the basis function. Remember that biorthogonal wavelet involves four basis function. They are scaling and wavelet functions at decomposition and reconstruction. When the coefficients of spline function are used as coefficients of one of the basis function, another basis function's coefficients can also be found from orthogonality. Then, from the properties the intended wavelet should possess gives sufficient number of relations, solving which the remaining basis functions can be formed. Wavelets based on standard spline function were designed and used on image compression with different wavelet based image coding schemes like EZW, SPIHT, STW, WDR and ASWDR. The coefficients from standard spline function follows that of binomial coefficients. Now, a modification is proposed by setting the centre coefficients a more weight than that in spline function. The modified spline is shown below.

$$
\begin{aligned}
& p_{i}=\frac{i+1}{2^{k-1}}\left(\begin{array}{c}
k \\
i
\end{array}\right) \text { where } \mathrm{i} \leq(\mathrm{k} / 2) \text { and } \\
& p_{i}=\frac{k-i+1}{2^{k-1}}\left(\begin{array}{l}
k \\
i
\end{array}\right) \text { where } \mathrm{i}>(\mathrm{k} / 2)
\end{aligned}
$$

Using this modification two new biorthogonal wavelets were defined by taking different lengths. For instance consider the compression performance with ASWDR using the biorthogonal wavelets with standard spline function and modified spline function as given in Table II.

From the Table II, it is evident that the improvement in the performance is less. The input image set considered is the standard test images used frequently in different image processing tasks. The images are high contrast, good dynamic range and clear images. On these set of test images the performance of the new wavelets is nominal. But the same new wavelets show a remarkable compression results on a slight darker images. The results are given in Table III.

From Table III, it can be seen a real betterment in compression results as both compression ratio and PSNR got increased. The new wavelet 1 and 2 are of different length. The second wavelet is of more length than the first. When the length is increased it can be observed that the compression ratio got increased drastically and noticeable improvement is observed in PSNR. The weight modification to the center coefficients of spline function has resulted in better compression performance with same coding technique for darker images. This kind of correspondences need to be revealed, so that an adaptive wavelet mechanism can be developed. Also, this kind of correspondence need to be identified for different image processing tasks as well. 
TABLE II: Compression performance of Biorthogonal wavelet with Standard and modified Spline function

\begin{tabular}{|c|c|c|c|c|c|}
\hline & & BIORS - 1 & BIORS - 2 & NBIOR - 1 & NBIOR - 2 \\
\hline \multirow[b]{2}{*}{ Lena } & $\mathrm{CR}$ & 6.8 & 8.8 & 6.4 & 9.8 \\
\hline & PSNR & 32.2 & 27.8 & 30.7 & 27.8 \\
\hline \multirow{2}{*}{ Cameraman } & $\mathrm{CR}$ & 10.4 & 7.0 & 4.9 & 15.4 \\
\hline & PSNR & 30.3 & 22.1 & 25.8 & 27.6 \\
\hline \multirow{2}{*}{ CT } & $\mathrm{CR}$ & 16.8 & 1.5 & 24.7 & 22.9 \\
\hline & PSNR & 49.0 & 19.3 & 46.7 & 36.6 \\
\hline \multirow{2}{*}{ Pepper } & $\mathrm{CR}$ & 7.7 & 17.1 & 7.9 & 12.4 \\
\hline & PSNR & 31.5 & 17.1 & 30.2 & 27.6 \\
\hline \multirow[b]{2}{*}{ Rice } & $\mathrm{CR}$ & 7.7 & 5.2 & 7.8 & 12.1 \\
\hline & PSNR & 32.9 & 24.9 & 30.4 & 27.2 \\
\hline \multirow[b]{2}{*}{ Barbara } & $\mathrm{CR}$ & 9.6 & 1.3 & 9.8 & 15.4 \\
\hline & PSNR & 29.6 & 14.2 & 28.6 & 25.4 \\
\hline \multirow[b]{2}{*}{ Mandrill } & $\mathrm{CR}$ & 5.4 & 5.4 & 13.3 & 5.3 \\
\hline & PSNR & 24.2 & 17.9 & 26.6 & 21.5 \\
\hline \multirow{2}{*}{ MRI } & $\mathrm{CR}$ & 11.9 & 3.2 & 22.5 & 20.8 \\
\hline & PSNR & 42.6 & 27.4 & 46.2 & 37.9 \\
\hline
\end{tabular}

TABLE III: Compression performance of Biorthogonal wavelet with Standard and modified Spline function on dark

\begin{tabular}{llcccc}
\multicolumn{5}{c}{ images } \\
\hline Image & & BIORS - 1 & BIORS - 2 & NBIOR - 1 & NBIOR - 2 \\
$\mathbf{1}$ & CR & 5.6 & 3.9 & $\mathbf{1 0 . 5}$ & $\mathbf{7 . 6}$ \\
& PSNR & 26.9 & 19.7 & $\mathbf{2 8 . 7}$ & $\mathbf{2 2 . 4}$ \\
$\mathbf{2}$ & CR & 13.3 & 2.8 & $\mathbf{2 5 . 8}$ & $\mathbf{1 8 . 7}$ \\
& PSNR & 29.1 & 15.7 & $\mathbf{3 3 . 2}$ & $\mathbf{2 7 . 2}$ \\
$\mathbf{3}$ & CR & 10.4 & 14.3 & $\mathbf{2 4 . 0}$ & $\mathbf{1 3 . 1}$ \\
& PSNR & 22.4 & 18.1 & $\mathbf{2 6 . 2}$ & $\mathbf{2 0 . 2}$ \\
$\mathbf{4}$ & CR & 7.1 & 5.6 & $\mathbf{1 3 . 9}$ & $\mathbf{9 . 1}$ \\
& PSNR & 31.1 & 23.9 & $\mathbf{3 3 . 6}$ & $\mathbf{2 8 . 2}$ \\
$\mathbf{5}$ & CR & 8.5 & 1.3 & $\mathbf{1 5 . 4}$ & $\mathbf{2 0 . 3}$ \\
& PSNR & 38.1 & 20.1 & $\mathbf{4 1 . 5}$ & $\mathbf{3 9 . 9}$ \\
$\mathbf{6}$ & CR & 27.1 & 4.4 & $\mathbf{3 9 . 0}$ & $\mathbf{5 0 . 2}$ \\
& PSNR & 47.3 & 26.4 & $\mathbf{4 9 . 5}$ & $\mathbf{4 4 . 9}$ \\
\hline
\end{tabular}

\section{Conclusions}

Wavelet based image compression schemes like EZW and SPIHT codes the wavelet coefficients in different manners so as to yield better compression performance. The accomplishment of any wavelet technique depends on the nature of wavelet coefficients resulted from the wavelet decomposition. To get good compression performance the coefficients should be formed in such a way that they can be coded easily and effectively. To get that sort of coefficients the basis function should be chosen properly. The basis function has to satisfy certain properties. This paper deals the basic properties of wavelets in terms of basis functions. Different basis functions along with important factors to consider in choosing a basis function are presented. The need of adaptive basis function selection is mentioned. 


\section{References}

[1] Kamrul Hasan Talukder, Koichi Harada, "A Scheme of Wavelet Based Compression of 2D Image", Proc. IMECS, Hong Kong, pp. 531-536, June 2006.

[2] Ahmed, N., Natarajan, T., and Rao, K. R., "Discrete Cosine Transform", IEEE Trans. Computers, vol. C-23, Jan. 1974, pp. 90-93, 10.1109/T-C.1974.223784.

[3] Pennebaker, W. B. and Mitchell, J. L. "JPEG Still Image Data Compression Standards", Van Nostrand Reinhold, 1993.

[4] Rao, K. R. and Yip, P., "Discrete Cosine Transform - Algorithms, Advantages, Applications", Academic Press, 1990, ISBN:0-12-580203-X.

[5] Wallace, G. K., "The JPEG Still Picture Compression Standard”, Comm. ACM, vol. 34, no. 4, April 1991, pp. 30-44, 10.1145/103085.103089.

[6] Eric J. Stollnitz, Tony D. Derose and David H. Salesin, "Wavelets for Computer Graphics- Theory and Applications", Morgan Kaufmann Publishers, Inc. San Francisco, California, ISBN-13: 9781558603752, ISBN-10: 1558603751.

[7] Vetterli, M. and Kovacevic, J., "Wavelets and Subband Coding", Englewood Cliffs, NJ, Prentice Hall, 1995, ISBN-13: 978-0130970800, ISBN-10: 9780130970800.

[8] G. K. Kharate, V. H. Patil, N. L. Bhale, "Selection of Mother Wavelet for Image Compression on Basis of Nature of Image", Journal of Multimedia, Academy Publisher, vol. 2, no. 6, November 2007, doi: 10.4304/jmm.2.6.44-51.

[9] Rubeena Vohra, Aakash Tayal, "Image Compression Based on Selection of Mother Wavelet", Proceedings of the 5th National Conference; INDIACom-2011, Computing For Nation Development, March 10 - 11, 2011, doi: 10.5121/ijma.2014.6206.

[10] J. Rafiee, M.A. Rafiee, N. Prause, M.P. Schoen, "Wavelet basis functions in biomedical signal processing", Expert Systems with Applications, ELSEVIER, 38, pp. 6190-6201, 2011, doi: 10.1016/j.eswa.2010.11.050.

[11] Sheikh Md. Rabiul Islam, Xu Huang, Kim Le, "MR Image Compression Based On Selection Of Mother Wavelet And Lifting Based Wavelet", The International Journal of Multimedia \& Its Applications (IJMA), Vol.6, No.2, April 2014.

[12] S. Grace Chang, Bin Yu, Martin Vetterli, "Adaptive Wavelet Thresholding for Image Denoising and Compression", IEEE Transactions on Image Processing, vol. 9, no. 9, September 2000, doi: $10.1109 / 83.862633$.

[13] François G. Meyer, Amir Z. Averbuch, Jan-Olov Strömberg, "Fast Adaptive Wavelet Packet Image Compression", IEEE Transactions on Image Processing, vol. 9, no. 5, May 2000, doi: $10.1109 / 83.841526$.

[14] Nasir M. Rajpoot, Roland G. Wilson, François G. Meyer, Ronald R. Coifman, “Adaptive Wavelet Packet Basis Selection for Zerotree Image Coding", IEEE Transactions on Image Processing, vol. 12, no. 12, December 2003, doi: 10.1109/TIP.2003.818115.

[15] Ronald A. DeVore, “Adaptive Wavelet Bases for Image Compression”, Wavelets Images and Surface Fitting, A K Peters, Wellesley, MA, ISBN 1-56881-040-7. 1994.

[16] Chuo-Ling Chang, Bernd Girod, "Direction-Adaptive Discrete Wavelet Transform for Image Compression", IEEE Transactions on Image Processing, vol. 16, no. 5, May 2007, doi: 10.1109/TIP.2007.894242.

[17] Shapiro J.M., "Embedded image coding using zerotrees of wavelet coefficients", IEEE Trans. Signal Proc., Vol. 41, No. 12, pp. 3445-3462, 1993, doi: 10.1109/83.784429.

[18] Wallace, "The JPEG still picture compression standard”, IEEE Transactions on Consumer Electronics, 38, 1992, doi: 10.1109/30.125072.

[19] Amir Said, William A. Pearlman, "An Image Multiresolution Representation for Lossless and Lossy Compression”, IEEE Trans. on Image Processing, Nov. 1993, doi: 10.1109/83.535842. 
[20] Walker J.S., "Wavelet-based Image Compression", Transforms and Data Compression Handbook, CRC Press LLC, Boca Raton, 2001.

[21] Tian J., Wells R.O. Jr., "Image Data Processing in the Compressed Wavelet Domain", 3rd International Conference on Signal Processing Proc., B. Yuan and X. Tang, (Eds.), 978-981, Beijing, China, 1996, doi: 10.1109/ICSIGP.1996.566254.

[22] Walker J.S., Nguyen T.O., "Adaptive Scanning Methods for Wavelet Difference Reduction in Lossy Image Compression", Proceedings of IEEE International Conference on Image Processing, 3, 182-185, 2000, doi: 10.1109/ICIP.2000.899325.

[23] Nill. N, "A Visual Model Weighted Cosine Transform for Image Compression and Quality Assessment", IEEE Transactions on Communications, vol.33, Issue 6, pp. 551 - 557, Jun 1985, doi: 10.1109/TCOM.1985.1096337.

[24] Sergio D. Servetto, Kannan Ramchandran, Michael T. Orchard, "Image coding based on a morphological representation of wavelet data", IEEE Trans. Image Processing 8(9): 1161-1174, 1999, doi: $10.1109 / 83.784429$.

[25] Bushra Al-Abudi and Loay George, "Color Image Compression Using Wavelet Transform", GVIP Conference, 19-21 Dec 2005, CICC, Cairo, Egypt

[26] Kamrul Hasan Talukder and Koichi Harada, "Haar Wavelet Based Approach for Image Compression and Quality Assessment of Compressed Image", IAENG International Journal of Applied Mathematics, 36:1, IJAM, 36(1), Feb 2007,

[27] V. Bruni, B. Piccoli, and D. Vitulano, "Wavelets and partial differential equations for image denoising", ELCVIA: Electronic Letters on Computer Vision and Image Analysis, 6.2 (2007): 36-53, doi: 10.5565/rev/elcvia.147.

[28] Mankar, V. H., et al. "Redundant Wavelet Watermarking using Spread Spectrum Modulation", ELCVIA Electronic Letters on Computer Vision and Image Analysis 7.2 (2008): 1-10, doi: 10.5565/rev/elcvia.267.

[29] Raja S.P, Suruliandi A., "Analysis of efficient wavelet based image compression techniques", IEEE International Conference on Computing Communication and Networking Technologies, pp. 1 - 6, July 2010, doi: 10.1109/ICCCNT.2010.5591634.

[30] Chithra, P.L. and Thangavel, P., "A fast and efficient memory image codec (encoding/decoding) based on all level curvelet transform coefficients with SPIHT and Run Length Encoding", IEEE Recent Advances in Space Technology Services and Climate Change, pp. 174 - 178, Nov. 2010.

[31] Jain, C., Chaudhary. V., Jain.K. and Karsoliya. S., "Performance analysis of integer wavelet transform for image compression", IEEE International Conference on Electronics Computer Technology, vol. 3, pp. 244 - 246, April 2011, doi: 10.1109/ICECTECH.2011.5941746.

[32] Li Wenna, Gao Yang, Yi Yufeng and Gao Liquun, "Medical image coding based on wavelet transform and distributed arithmetic coding", IEEE Chinese Control and Decision Conference, pp. 4159 - 4162, May 2011, doi: 10.1109/CCDC.2011.5968955.

[33] Xuechun Wang, Shenxiao Liu and Fang Han, "A fast image coding algorithm based on lifting wavelet transform", International Congress on Image and Signal Processing, vol. 2, pp. 594 - 597, Oct. 2011, doi: 10.1109/CISP.2011.6100373.

[34] PE Leni, YD Fougerolle, F Truchetet, "Kolmogorov superposition theorem for image compression", IET Image Processing, vol. 6, no. 8, pp. 1114-1123, 2012, doi: 10.1049/iet-ipr.2011.0459.

[35] Hong-jun Li, Zheng-guang Xie, Wei Hu, "An image compression method using sparse representation and grey relation", Proceedings of 2013 IEEE International Conference on Grey systems and Intelligent Services (GSIS), 15-17 Nov 2013, doi: 10.1109/GSIS.2013.6714741.

[36] Jaya Krishna Sunkara, E Navaneethasagari, D Pradeep, E Naga Chaithanya, D Pavani, D V Sai Sudheer, "A New Video Compression Method using DCT/DWT and SPIHT based on Accordion Representation", I.J. Image, Graphics and Signal Processing, pp. 28-34, May 2012, doi: 10.5815/ijigsp.2012.04.04. 
[37] Naqvi, S.A.R, Touqir, I, Raza, S.A., "Adaptive geometric wavelet transform based two dimensional data compression", IEEE GCC Conference and Exhibition, pp. 583 - 588, Nov. 2013, doi: 10.1109/IEEEGCC.2013.6705844.

[38] Mulla. A, Baviskar. J, Wagh. S, Kudu. N, Baviskar. A, "Probabilistic triangular shuffling approach in DWT based image compression scheme", IEEE International Conference on Communication, Information \& Computing Technology (ICCICT), pp. 1-6, Jan 2015, doi: 10.1109/ICCICT.2015.7045658.

[39] Paul A, Khan T.Z, Podder P, Ahmed R, Rahman M.M, Khan M.H, "Iris image compression using wavelets transform coding", IEEE 2015 2nd International Conference on Signal Processing and Integrated Networks (SPIN), pp. 544-548, Feb 2015, doi: 10.1109/SPIN.2015.7095407.

[40] Andreas Weinlich, Peter Amon, Andreas Hutter, Andre Kaup, "Probability Distribution Estimation for Autoregressive Pixel-Predictive Image Coding”, IEEE Trans. on Image Processing, vol. 25, Iss. 3, pp. 1382-1395, March 2016, doi: 10.1109/TIP.2016.2522339.

[41] Miguel Hernandez-Cabronero, Ian Blanes, Armando J. Pinho, Micheal W. Marcellin, Joan SerraSagristà, "Progressive Lossy-to-Lossless Compression of DNA Microarray Images", IEEE Signal Processing Letters, Vol.23, Iss.5, pp. 698-702, May 2016, doi: 10.1109/LSP.2016.2547893.

[42] Miguel Hernandez-Cabronero, Ian Blanes, Armando J. Pinho, Micheal W. Marcellin, Joan SerraSagristà, "Analysis-Driven Lossy Compression of DNA Microarray Images", IEEE Transactions on Medical Imaging, vol.35, Iss.2, pp. 654-664, Feb 2016, doi: 10.1109/TMI.2015.2489262.

[43] Karthik, R., and R. Menaka. "A critical appraisal on wavelet based features from brain MR images for efficient characterization of ischemic stroke injuries", ELCVIA: Electronic Letters on Computer Vision and Image Analysis 15.3 (2016): 1-16, doi: 10.5565/rev/elcvia.804.

[44] C. Yang, Y. Zhao and S. Wang, "Deep image compression in the wavelet transform domain based on high frequency sub-band prediction," in IEEE Access. doi: 10.1109/ACCESS.2019.2911403.

[45] Jaya Krishna Sunkara, Purnima Kuruma, Ravi Sankaraiah Y, "Image Compression Using Hand Designed and Lifting Based Wavelet Transforms", International Journal of Electronics Communications and Computer Technology, vol. 2 (4), 2012.

[46] Jaya Krishna Sunkara, K Subramanyam, V. V. Satyanarayana Tallapragada, V Kiran Kumar, "Image Compression by SPIHT Coding using Traditional Wavelets", Journal of Emerging Technologies and Innovative Research (JETIR), vol. 5, issue 5, pp. 256-267, May 2018.

[47] H. Caglar, Y. Liu, and A. N. Akansu, "Statistically optimized PR-QMF design", Proc. of SPIE : Visual Communications and Image Processing 1605, pp. 86-94, 1991, doi: 10.1117/12.50231.

[48] A. H. Tewfik, D. Sinha and P. Jorgensen, "On the optimal choice of a wavelet for signal representation", IEEE Trans. on Information Theory 38(2), 747-765, March 1992, doi: $10.1109 / 18.119734$.

[49] K. Ramchandran and M. Vetterli, "Best wavelet packet in a rate-distortion sense", IEEE Trans. on Image Processing 2(2), 160-175, April 1993.

[50] A. Kohen, I. Daubechies, J.C. Feauveau, "Biorthogonal bases of compactly supported wavelets", Comm. Pur. \& Appl. Math., 1991, doi: 10.1002/cpa.3160450502.

[51] S. Mallat, "Multiresolution approximations and wavelet orthogonal bases of $\mathrm{L}^{2}(\mathrm{R})$ ", Transactions of American Mathematics Society, vol. 315, no. 1, pp. 69-87, 1989.

[52] I. Daubechies, "Orthogonal bases of compactly supported wavelets", Comm. Pur. \& Appl. Math., vol. 41, pp. 909-996, 1988, doi: 10.1002/cpa.3160410705.

[53] A. S. Lewis, G. Knowles, "Image compression using the 2D wavelet transform", IEEE Trans. on image processing, vol. I, pp. 244-250, Feb 1992.

[54] Ronald A. DeVore, "Adaptive Wavelet Bases for Image Compression”, A K Peters Wavelets, Images and Surface Fitting, pp. 197-219, 1994.

[55] Yan Zhuang, John S. Baras, "Image compression using optimal wavelet basis", Proc. of SPIE, 17-21 April 1995, doi: 10.1117/12.205380. 
[56] Michael G. Strintzis, "Optimal Biorthogonal Wavelet Bases for Signal Decomposition”, IEEE Transactions on Signal Processing, vol. 44, no. 6, pp. 1406-1417, June 1996, doi: 10.1109/78.506607.

[57] Aleksandra Mojsilovic, Miodrag V. Popovic and Dejan M. Rackov, "On the Selection of an Optimal Wavelet Basis for Texture Characterization”, IEEE Transactions on Image Processing, vol. 9, no. 12, pp. 2043-2050, Dec 2000, doi: 10.1109/83.887972.

[58] Nasir M. Rajpoot, Roland G. Wilson, Francois G. Meyer, Ronald R. Coifman, "Adaptive Wavelet Packet Basis Selection for Zerotree Image Coding", IEEE Transactions on Image Processing, vol. 12, no. 12, Dec 2003, doi: 10.1109/TIP.2003.818115.

[59] M. K. Mandal, S. Panchanathan and T. Aboulnasr, "Choice of Wavelets for Image Compression", Springer Lecture Notes in Comp. Science, vol. 1133, pp. 239-249, 2005, doi: 10.1007/BFb0025147.

[60] G. K. Kharate, V. H. Patil and N. L. Bhale, "Selection of mother wavelet for image compression on basis of image", Journal of Multimedia, vol. 2, no. 6, November 2007, doi: 10.1109/ICSCN.2007.350747.

[61] Maria Rehman, Imran Touqir, Wajiha Batool, "Selection of optimal wavelet bases for image compression using SPIHT algorithm", Proc. SPIE 9445, Seventh International Conference on Machine Vision (ICMV 2014), vol. 9445, Feb 2015, doi: 10.1117/12.2182135.

[62] Noor Kamal Al-Qazzaz, Sawal Hamid Bin Mohd Ali, Siti Anom Ahmad, Mohd Shabiul Islam and Javier Escudero, "Selection of Mother Wavelet Functions for Multi-Channel EEG Signal Analysis during a Working Memory Task", Sensors, 15, pp. 29015-29035, 2015, doi: 10.3390/s151129015.

[63] Girisha Garg, "A signal invariant wavelet function selection algorithm", Springer Medical \& Biological Engineering Computing, 54, pp. 629-642, 2016, doi: 10.1007/s11517-015-1354-z.

[64] Jiajia Liu, W.H. Siew, John J. Soraghan and Euan A. Morris, "A Novel Wavelet Selection Scheme for Partial Discharge Signal Detection under Low SNR Condition," 2018 IEEE Conference on Electrical Insulation and Dielectric Phenomena, Mexico, 21-24 Oct. 2018, doi: 10.1109/CEIDP.2018.8544802.

[65] Sushree Satvatee Swain and Dipti Patra, "Multiscale energy based suitable wavelet selection for detection of myocardial infarction in ECG," Healthcare Technology Letters, vol. 6, issue 1, 2019, doi: 10.1049/htl.2018.5007. 